\title{
CRITIQUE THEORIQUE
}

\section{ET DIFFERENCE SOCIALE}

Ceux qui pensent que la structure sociale, politique et culturelle de notre monde actuel est la seule possible, ceux-là déplorent tout de même, en général, cette impossibilité de faire mieux. D'autres, moins nombreux, touchés par l'immense souffrance des hommes, protestent contre l'état actuel des choses et déploient des efforts d'amélioration.

Les uns et les autres, pour absoudre ou pour condamner, se réferent à des principes moraux supérieurs, ou, plus simplement à la morale en général. Leur point de vue commun, c'est que, dans la vie sociale et politique, on devrait s'inspirer de ces principes moraux, de cette morale. Ils commandent ou ils obligent. Si l'on veut améliorer le monde humain, "il n'y a qu'à" appliquer des maximes morales universellement connues et admises.

La théorie met en évidence le contraste entre les faits et l'idéal, et, louablement, elle réclame une mise en conformité. Elle promulgue des lois imperatives. Ainsi, par exemple, Kant déclare-t-il, entre autres maximes: "Agis de telle sorte que tu traites toujours l'humanité aussi bien dans ta personne que dans la personne de tout autre toujours en même temps comme une fin, et jamais simplement comme un moyen ${ }^{n 1}$.

Les revendications morales d'universalité, de justice, de charité portent fréquemmentsur des différences sociales tenues pour inhumaines, ou incohérentes. Il ne s'agit pas d'abolir les différences individuelles, souhaitables et, en tout cas, inéliminables. Ce sont les différences sociales qui sont visées, celles qui tiennent à la structure des sociétés et qui, précisément, empêchent le développement des différences individuelles, gênent l'épanouissement libre et généreux des individus, en excluant aussi toute harmonie globale.

Ainsi, dans l'Antiquité, se trouvaient par exemple distinguées et opposées des catégories sociales typiques: Grec et Barbare, maître et esclave. Plus tard se différencient les suzerains et les vassaux, les seigneurs et les serfs, les nobles et les roturiers, etc.

\footnotetext{
${ }^{1}$ Kant, Fondements de la metaphysique des moeurs, trad. par V. Delbos. Paris: Delagrave, 1959, pp. 150-151.
} 
De telles differences sociales ont subi une critique morale renouvelée, à l'occasion de la célébration récente du bicentenaire de la Révolution française. Celle-ci se présente d'ailleurs souvent et parfois exclusivement, comme le resultat de la critique morale effectué par les philosophes du XVIIIè siècle à l'égard de l'Ancien régime.

Et effectivement, cette critique multiforme et incisive a joué un rôle dans les événements. On ne saurait contester son efficacité, tout en remarquant qu'elle a évidemment contribué à éliminer les vestiges du passé plus qu'elle n'a guidé la construction et l'invention de l'avenir.

Toutefois, l'observation et l'expérience montrent assez que cette critique morale reste souvent et longtemps lettre morte. Pourquoi donc les droits de l'homme ne se sont-ils accomplis, et précairement, qu'après des millenaires d'histoire humaine? On en vient à s'interroger sur les conditions d'apparition et de réussite de la revendication d'harmonisation et d'unification de la vie sociale. Quand et comment les différences sociales moralement réprouvées, peuvent-elles être effectivement effacées?

De nombreux exemples tendent à montrer que cette opération ne s'effectue que lorsq ue des conditions sociales objectives la permettent. Les impératifs moraux ne jouissent pas d'une puissance absolue.

Par souci de briéveté, tenons-nous en, pour le montrer, au seul exemple de l'abolition de l'esclavage. Le conditionnement social des condamnations morales de l'esclavege, différence sociale abrupte, ont été mises en evidence par Tooqueville, entre autres analystes perspicaces.

Dans un chapitre remarquable de La démocratie en Amérique, il montre, après toutes sortes de constatations, de comparaisons et de réflexions, et après avoir signalé la cherté de l'achat et de l'entretien des esclaves africains, en même temps que leur faible productivité, que les maîtres ont intérêt à se débarrasser de leurs esclaves et à les remplacer par des travailleurs libres salariés: "L'ouvrier libre est payé, mais il fait plus vite que l'esclave, et la rapidité de l'exécution est un des grands éléments de l'économie. Le blanc vend ses secours, mais on ne les achète que lorsqu'ils sont utiles; le noir n'a rien à réclamer pour prix de ses services, mais on est obligé de le nourrir en tout temps." Tooqueville poursuit longuement ce raisonnement qui le conduit à la conclusion, d'ailleurs évidente, qu'en réalité l'esclave a plus coûté que l'homme libre, et ses travaux ont été moins productifs"

${ }^{2}$ A de Tocqueville. De la démocrative en Amerique. Paris: Garnier-Flammarion, 1981, I, p. 461. 
Il reste toutefois aveugle sur le fait qu'il n'en a pas toujours été ainsi, et que, pendant une longue période historique, l'esclavage a été rentable, et hautement, sans quoi il ne se serait pas maintenu. Il n'en est pas moins amené à constater: "Ce n'est pas dans l'intérêt des nègres, mais dans celui des blancs, qu'on détruit l'esclavage aux Etats-Unis"3.

A bien des égards, le libéralisme, économique et social, repose sur de telles constatations concernant la structure et le fonctionnement du monde moderne. Adam Smith l'avait déjà proclamé (1776): "l'expérience de tous les temps et de tous les pays s'accorde, je crois, pour démontrer que l'ouvrage fait par des mains libres, revient à la fin à meilleur compte que celui qui est fait par des esclaves" ${ }^{\text {n. }}$.

Reste toutefois à expliquer pourquoi les hommes sont restés si longtemps imperméables à "l'expérience de tous les temps et de tous les pays"!

En réalité, beaucoup d'observateurs remarquent, au début du XIXè siècle, que les conditions économiques et techniques ont changé, et que c'est cela qui rend l'esclavage inutile et même ruineux pour les maîtres eux-mêmes, par exemple dans les colonies françaises.

Des publicistes décrivent alors l'horreur de l'esclavage, à juste titre, mais ils le font d'autant plus volontiers qu'ils démontrent en même temps son manque de rentabilité.

On lit dans certaines publications d'horribles calculs comparatifs du prix de revient d'un esclave et d'un salarié libre, et ces calculs dénoncent, mieux que toutes les lamentations morales, l'atrocité du système esclavagiste, jusque dans ses détails.

Pourtant il persiste encore, à cette époque, et il a fallu attendre pour qu'il soit remplacé completement par une difference sociale d'un nouveau type.

Il avait pourtant été réprouvé dès longtemps au nom de principes supérieurs. Curieusement, certains grands esprits semblaient ne même pas se rendre compte du contraste entre leurs proclamations idéales et la réalité tolérée ou renforcée.

Ainsi, Tocqueville lui-même déclarait, dans le chapitre déjà évoqué, et en contradiction avec les constats qu'ils avait dressés, que "le christianisme a détruit l'esclavage en faisant valoir les droits des esclaves", ce qui ne l'empéchait pas d'ajouter, plus lucidement, reconnaissantqu'en fait l'esclavage n'avait pas du tout été "détruit": "de nos jours on peut

${ }^{3}$ Ibid., p. 458.

${ }^{4}$ A Smith. Recherches sur la nature et les causes de la richesse des nations. Trad. par G. Garnier. Pasris: 1801, tome I, p. 165. 
attaquer l'esclavage au nom du maître: sur ce point l'intérêt et la morale sont d'accord".

C'est la conclusion à laquelle parvenaient bien d'autres observateurs, et que l'un d'eux exprima ainsi: "dans le siècle où nous vivons, et avec la nature des idées qui nous dominent, je me suis félicité de pouvoir démontrer que les calculs étroits de l'égoïsme, de l'avarice et de la cupidité, doivent être d'accord s'ils sont éclairés sur leurs véritables intérêts, avec les principes élevés et invariables de la morale, de la justice et de la charitens.

On ne saurait mieux reconnaître que l'application ou la réalisation des principes soi-disant invariables de la morale, dépend de la variation historique des conditions sociales. On peut s'étonner du fait que les auteurs qui parvinrent à cette conclusion ne crurent pas devoir analyser cette variation elle-même et ses causes éventuelles, et regretter qu'ils n'aient pas appliqué la même méthode d'observation à tous les épisodes hsitoriques de la variation sociale. Ils auraient vu plus nettement encore que la morale ne peut pas plus que la connaissance se séparer de la réalité objective et s'opposer abruptement à elle: une colombe ne saurait voler sans la résistance de l'air.

C'est ce qu'affirmait Pierre Leroux en 1827, au beau milieu de la querelle de l'abolition de l'esclavage: "La notion du droit en cette matière a suivi les progrès de la vie sociale. Tant que l'esclavage n'a pas été directement contraire à l'essence de la société, on ne l'as pas traité de crime. C'est que les speculations des philosophes ont beau être hardies, elles ont toujours leurs racines dans leur siecle: ils ont beau s'isoler et s'abstraire, c'est toujours le monde de leur temps qui leur donne l'impulsion" ${ }^{\text {" }}$

s Dureau de Lamalle. "Mémoire", in Le Globe, V, No 3, 19 Aoot 1826, p. 16.

${ }^{6}$ Le Globe, IV, No. 76, 6 Février 1827, vol.I p. 402. 